\begin{tabular}{|c|c|}
\hline Title & A ustenite Grain Growth in Peritectic Solidified Carbon Steels A nalyzed by Phase-Field Simulation \\
\hline Author(s) & Ohno, Munekazu; Tsuchiya, Shingo; Matsuura, Kiyotaka \\
\hline Citation & $\begin{array}{l}\text { Metallurgical and Materials T ransactions A , 43(6), 2031-2042 } \\
\text { https://doi.org/10.1007/s11661-011-1072-6 }\end{array}$ \\
\hline Issue Date & $2012-06$ \\
\hline Doc URL & http://hdl.handle.net/2115/49354 \\
\hline Rights & The final publication is available at www.springerlink.com \\
\hline Type & article (author version) \\
\hline File Information & MMTA 43-6_2031-2042.pdf \\
\hline
\end{tabular}

Instructions for use 


\title{
Austenite grain growth in peritectic solidified carbon steels analyzed by phase field simulation
}

\author{
Munekazu Ohno, Shingo Tsuchiya and Kiyotaka Matsuura
}

\begin{abstract}
Formation of coarse columnar grains (CCG) in as-cast austenite structure of peritectic carbon steels is one of the serious problems in continuous casting processes. It was recently elucidated that the formation of CCG is ascribed to a discontinuous grain growth. Furthermore, the critical condition for the discontinuous growth to occur was elicited on the basis of phase field simulations and a theory of grain growth. In this study, by means of the phase field simulations, the detailed investigation is carried out for grain coarsening of as-cast austenite structure. It is demonstrated in the two-dimensional simulations that the coarsest grain structure emerges by the discontinuous growth in the vicinity of the critical condition. In addition, a model for predicting the upper limit of grain size during the discontinuous growth is proposed. The model successfully describes the experimental result with reasonable accuracy.
\end{abstract}




\section{INTRODUCION}

As-cast $\gamma$-austenite structure in continuously cast slabs of peritectic carbon steels consists of coarse columnar grains $(\mathrm{CCG})$ in the vicinity of the slab surfaces. Since the size of CCG is closely related to the occurrence of surface cracking and low ductility of the slabs, ${ }^{[1-4]}$ the precise description and prediction of the as-cast $\gamma$ grain size are quite important issues in the field of casting of steels.

A number of attempts have been made for the description and prediction of size of the CCG during continuous casting processes. ${ }^{[5-10]}$ In the early works, the CCG structure has been supposed to originate from continuous grain growth which occurs during cooling below a temperature for completion of transformation to $\gamma$ single phase, $T_{\gamma}{ }^{[5-8]}$ However, our recent study by means of rapid unidirectional solidification experiment revealed that the formation of CCG structure is actually ascribed to a discontinuous grain growth. ${ }^{[1]}$ Figure 1 shows the as-cast $\gamma$ grain structure observed in a 0.2 mass $\%$ carbon steel quenched during the unidirectional solidification. The CCG develop from the mold side to the upper part. Importantly, fine columnar $\gamma$ grains (FCG) exist ahead of the CCG region. The boundary between FCG and CCG regions, which in this paper is termed the FCG/CCG region boundary (FCRB), is identified in Figure 1. From temperature measurements, it was found that the temperature at FCRB is always $T_{\gamma}$ during the solidification and, hence, the FCG region corresponds to liquid $+\gamma$ two phase field, while the CCG region is $\gamma$ single phase field. 
Although the FCG always form ahead of the growing CCG during the solidification, the FCG near FCRB shrink with the growth of CCG. More specifically, coarse $\gamma$ grains, which formed in the vicinity of the mold wall, preferentially develop along the temperature gradient below $T_{\gamma}$ at the expense of FCG, finally growing to CCG. This process is characterized by vertical motion of FCRB in Figure 1 and is classified as a discontinuous grain growth. Since the liquid phase in FCG region prevents the migration of FCRB, the FCRB moves upward together with the position of $T_{\gamma}$.

In our recent study, ${ }^{[12]}$ the occurrence of the discontinuous growth in various cooling conditions and the CCG formation by this mechanism were verified by means of phase field simulations. Then, we elicited the critical condition for the discontinuous growth to start and continue based on a theory of grain growth, which is given by, ${ }^{[12]}$

$$
V_{\mathrm{T}} \leq M^{\prime}\left(T_{\gamma}^{*}\right) \frac{1}{\xi d_{\text {initial }}}
$$

where $V_{\mathrm{T}}$ is the migration velocity of $T_{\gamma}$ position called the thermal velocity in this paper, $M^{\prime}\left(T_{\gamma}^{*}\right)$ is the grain boundary mobility at a temperature near FCRB, $T_{\gamma}^{*}$, which is given by $T_{\gamma}{ }^{*}=T_{\gamma}-G \xi d_{\text {initial }}$ with a temperature gradient, $G . d_{\text {initial }}$ is the short axis diameter of FCG and $\xi$ is a parameter determined by the local shape of FCRB; $\xi=1$ for two-dimensional (2-D) system and $\xi \approx 0.78$ for three-dimensional (3-D) system. The right-hand side of Eq. [1] corresponds to the migration velocity of FCRB, which is denoted as $V_{\text {critical }}$ in this paper. The structure map constructed from Eq. [1] is exemplified for $\xi=1, T_{\gamma}=1743 \mathrm{~K}$ and $d_{\text {initial }}=150$ 
$\mu \mathrm{m}$ in Figure 2(a). This map specifies the range of cooling conditions for the discontinuous growth. This map should be useful in predicting CCG formation in continuously cast slabs in practice.

Since the focus in our recent paper $^{[12]}$ was directed at elucidation of the critical condition, details on the grain size in each growth condition were not discussed. In the early works,${ }^{[5-8]}$ the description and prediction of as-cast $\gamma$ grain size have been attempted based on the assumption that the CCG form by continuous grain growth during cooling below $T_{\gamma}$. One of the important findings in our previous experimental study ${ }^{[11]}$ is that the short axis diameter of CCG increases only near FCRB, viz., near $T_{\gamma}$ and it does not virtually change during cooling below $T_{\gamma}$. This fact is in marked contrast to the assumption made in early works. Therefore, it is indispensable for an appropriate description of size of the CCG to examine grain coarsening during the discontinuous growth in great detail and to revisit the conventional models in the light of the continuous and discontinuous growth processes. In the present paper, we first demonstrate effects of cooling conditions and material parameters on the as-cast $\gamma$ grain sizes obtained by 2 -D phase field simulations. It will be demonstrated that the coarsest grain structure emerges by the discontinuous growth in the vicinity of the critical condition. One will see that the conventional models based on the continuous growth fairly underestimate the size of CCG formed by the discontinuous growth. Then, we propose a model for predicting the coarsest grain size based on a theory of grain growth and 
demonstrate that this model successfully explains the experimental fact with reasonable accuracy.

\section{COMPUTATIONAL DETAILS}

The detail of the phase field model employed in this study is found in References 13 and 14 and the computational conditions are the same as those reported in our recent paper. ${ }^{[12]}$ Here we explain the computational system and the essential equations used in this study. As described in the introduction, FCG always exist ahead of the growing CCG. The FCG cannot grow along its short axis direction because of the pinning effect of liquid phase which also prevents the FCRB from moving. ${ }^{[1]}$ In the light of these facts, we omitted the initial formation process of FCG and we dealt with only the grain growth starting from already formed FCG structure during cooling as detailed below.

Within the multi-phase field model, ${ }^{[13,14]}$ grain growth in polycrystalline materials is described by the temporal evolution of phase field, $\phi_{i}(\mathbf{r}, t)$, which represents a probability of finding a grain with an orientation, $i$, at given spatial point, $\mathbf{r}$, and time, $t$. The temporal evolution of $\phi_{i}$ is described by the following equation, ${ }^{[14]}$

$$
\frac{\partial \phi_{i}}{\partial t}=-\frac{2 L(T)}{n} \sum_{j \neq i}^{N} s_{i} s_{j}\left[\frac{\varepsilon^{2}}{2}\left(\nabla^{2} \phi_{j}-\nabla^{2} \phi_{i}\right)+\omega\left(\phi_{j}-\phi_{i}\right)\right],
$$

where $L(T)$ is the phase field mobility of temperature dependent, $s_{i}$ is a step function with $s_{i}=$ 
1 for $\phi_{i}>0$ and $s_{i}=0$ otherwise and $n$ represents the number of phases existing at a given spatial point as defined by $n=\sum_{i}^{N} s_{i}$ with the total number of crystallographic orientations counted in the simulation, $N . \varepsilon$ and $\omega$ are the gradient energy coefficient and a height of parabolic potential, respectively. Within the phase field model, the grain boundary is described as the diffuse interface with a finite thickness, $W . \varepsilon$ and $\omega$ are associated with $W$ and the grain boundary energy, $\sigma$, as given by $\varepsilon=2 \sqrt{W \sigma} / \pi$ and $\omega=4 \sigma / W$. In the present study, $\sigma$ is set to $\sigma=0.79 \mathrm{~J} \mathrm{~m}^{-2}[15]$ and $W$ is given to be $W=6 \cdot \Delta x$ with the square grid spacing, $\Delta x=10^{-5} \mathrm{~m}$. The temperature dependence of $L(T)$ is defined as $L(T)=L_{0} \exp (-Q /(R T))$ with the gas constant, $R$. Here, $L_{0}$ and $Q$ were determined to be $L_{0}=104 \mathrm{~m}^{3} \mathrm{~J}^{-1} \mathrm{~s}^{-1}$ and $Q=177$ $\mathrm{kJ} \mathrm{mol}^{-1}$ based on the reported values of the grain boundary mobility in $\gamma$ phase. ${ }^{[7]}$ These values were chosen as typical values of steels and we do not focus on a specific composition in the phase field simulations. Also, the crystallographic anisotropies of $\sigma$ and the grain boundary mobility are not taken into account for simplicity.

The computational system is $2-\mathrm{D}$ system divided by $1000 \times 500$ square grid points for $x$ - and $y$-axes, respectively. The system size accordingly corresponds to $10 \mathrm{~mm} \times 5 \mathrm{~mm}$. As mentioned above, we focus on the grain growth from the FCG structure. Hence, the system initially consists of only FCG. Several types of initial FCG structures were obtained by preliminarily performing isothermal phase field simulations for different conditions. ${ }^{[12]}$ It was found in the experimental study ${ }^{[11]}$ that the short axis diameter of FCG is always equal to the 
primary dendrite arm spacing (DAS) during the solidification process, which indicates the strong pinning effect of liquid phase in FCG region. Because detailed descriptions of pinning phenomena on motion of FCRB requires large computational burdens, we used an effective mobility approach ${ }^{[16]}$ to take the strong pinning effect into account. Within this approach, the grains are not allowed to grow above $T_{\gamma}$. When the temperature decreases to $T_{\gamma}$, the grain growth starts at the corresponding point. The growth rate is then controlled by the phase field mobility of temperature dependent, $L(T) \cdot{ }^{[12]}$

A linear temperature gradient, $G$, was unidirectionally applied along $y$-direction. $G$ is constant with time, ranging from $G=2 \times 10^{2}$ to $5 \times 10^{6} \mathrm{~K} \mathrm{~m}^{-1}$. The cooling rate, $\dot{T}$, was set to a constant and it was varied from $\dot{T}=0.1$ to $50 \mathrm{~K} \mathrm{~s}^{-1}$. Then, the thermal velocity, $V_{\mathrm{T}}$, is given by $V_{\mathrm{T}}=\dot{T} / G$ which is equivalent to the migration velocity of $T_{\gamma}$ position. The periodic boundary condition and zero flux boundary condition for $\phi_{i}$ were applied to $x$ - and $y$-directions, respectively. The initial temperature was set above $T_{\gamma}$ over the whole system. $T_{\gamma}$ was chosen to be $T_{\gamma}=1743$ and $1643 \mathrm{~K}$. The lower edge of $y$-axis is the lowest temperature side at which the initial temperature is $T_{\gamma}$. Hence, the position of $T_{\gamma}$ moves from the lower to upper edge of $y$-axis during cooling. When $T_{\gamma}$ position reaches the upper edge of $y$-axis, the calculation was stopped.

The average grain diameters along $x$ - and $y$-directions were estimated by the linear intercept method. Since the columnar grains are elongated along $y$-direction in the present 
simulations, the diameter along $y$-axis ( $x$-axis) corresponds to long (short) axis diameter in columnar grain structures. For the sake of convenience, the grain diameters along $x$ - and $y$-axes are described as short axis diameter, $d_{\mathrm{S}}$, and long axis diameter, $d_{\mathrm{L}}$, respectively, even in the case of equiaxed grain structures. The aspect ratio is defined as the ratio of $d_{\mathrm{L}}$ to $d_{\mathrm{S}}$. We focused on several initial grain structures, i.e., FCG structures characterized by $a_{\text {initial }}=1,3$ and $d_{\text {initial }}=100,150$ and $200 \mu \mathrm{m}$. Here, $a_{\text {initial }}$ and $d_{\text {initial }}$ represent the initial aspect ratio and initial short axis diameter of FCG, respectively.

It is noted that the analyses and discussions in this paper are entirely based on the results of 2-D simulations. In the analysis of grain size, the system size should be made large enough to obtain statistically reliable results. Therefore, the analysis based on 3-D simulations is computationally demanding. Although 2-D simulations might fail to produce some important features which emerge only in 3-D system, the result of 2-D simulations provides a basis for an understanding of grain growth in 3-D system. In this paper, the discussions are presented with a careful attention paid to the difference between 2-D and 3-D processes.

\section{RESULTS AND DISCUSSION}

\section{A. Structure map for equiaxed and columnar grains}

The critical condition for the discontinuous growth to occur, which was the main concern in the previous study ${ }^{[12]}$, is briefly explained to promote better understanding of the 
subsequent discussions. Figure 2(a) is the structure map calculated for $T_{\gamma}=1743 \mathrm{~K}$. The solid line in Figure 2(a) is the calculated result for $V_{\mathrm{T}}=V_{\text {critical }}$ (Eq. [1]). Figures 2(b)-(g) show the microstructures during 2-D grain growth under the cooling condition specified in Figure 2(a). The lower part of each micrograph corresponds to the lowest temperature side, i.e., the mold side. The position of $T_{\gamma}$ moves from the lower to upper edge along $y$-direction and the grain growth accordingly starts from the lower part. These are the microstructures at a time when the position of $T_{\gamma}$ reaches half of total length of $y$-axis as indicated by the arrow on the right-hand side in each micrograph. The initial grain structures for Figures 2(b)-(d) are the equiaxed grain structure with $a_{\text {initial }}=1$ and $d_{\text {initial }}=150 \mu \mathrm{m}$, while those for Figures 2(e)-(g) are the columnar grain structure with $a_{\text {initial }}=3$ and $d_{\text {initial }}=150 \mu \mathrm{m}$. Note that the structure map shown in Figure 2(a) is independent of the value of $a_{\text {initial }}{ }^{[12]}$ When the cooling condition is located in the continuous growth region in Figure 2(a), the grain size changes continuously near $T_{\gamma}$ and the grain growth thereby takes place continuously from FCG as can be seen in Figures 2(d) and (f). On the other hand, in Figures 2(b), (c) and (e) whose cooling conditions are in the discontinuous growth region, the CCG develop and the FCRB is clearly identified. Although not shown here, the width of CCG gradually increases with the migration of FCRB. When $T_{\gamma}$ reaches the upper edge of $y$-axis, most of grains are elongated along $y$-direction. It should be mentioned that the aspect ratio, $a_{\mathrm{f}}$, in the final structure takes a higher value than $a_{\text {initial }}$ only when the discontinuous growth arises. ${ }^{[12]}$ In the case of Figure $2(\mathrm{~g})$, both the 
discontinuous and continuous processes hardly take place due to high $\dot{T}$ and large $G .^{[12]}$

As described above, the structure map in Figure 2(a) specifies the range of cooling conditions for discontinuous growth and, therefore, it indicates the shape of final grain structures, columnar or equiaxed shape. In the next section, our attention is directed at the final grain size in each growth condition. One will see that the final grain size largely depends on the type of grain growth mechanism, the cooling condition and the initial FCG structure.

\section{B. Effects of growth conditions on grain size}

In this section, dependences of grain sizes, $d_{\mathrm{L}}$ and $d_{\mathrm{S}}$, on $\dot{T}$ (thus $G$ ), $d_{\text {initial }}, T_{\gamma}$ and $a_{\text {initial }}$ are discussed. The calculated long axis diameter in the final structure, $d_{\mathrm{L}}$, is plotted with respect to the thermal velocity, $V_{\mathrm{T}}$, in Figure $3(\mathrm{a})$. These are the results for $T_{\gamma}=1743 \mathrm{~K}, d_{\text {initial }}$ $=150 \mu \mathrm{m}$ and $a_{\text {initial }}=3 . \dot{T}$ is fixed for each set of data. Hence, the increase in $V_{\mathrm{T}}$ corresponds to a decrease in $G$. The vertical dotted lines denote $V_{\text {critical }}$ for each value of $\dot{T}$. $V_{\text {critical }}$ is slightly lowered by increasing $\dot{T}$ because the larger value of $G$ results in lower values of $T_{\gamma}{ }^{*}$ and the mobility of FCRB (Eq. [1]). At $\dot{T}=1.0 \mathrm{~K} \mathrm{~s}^{-1}, d_{\mathrm{L}}$ slightly increases with decreasing $V_{\mathrm{T}}$ from $V_{\mathrm{T}}=5 \times 10^{-3} \mathrm{~m} \mathrm{~s}^{-1}$, which is because the grains are allowed to grow for a longer period of time as $V_{\mathrm{T}}$ becomes lower. When $V_{\mathrm{T}}$ falls below $V_{\text {critical }} \approx 1 \times 10^{-4} \mathrm{~m} \mathrm{~s}^{-1}, d_{\mathrm{L}}$ sharply rises to about $4 \mathrm{~mm}$, which indicates the occurrence of discontinuous grain growth. Similarly, the sharp rising of $d_{\mathrm{L}}$ appears at $\dot{T}=10$ and $20 \mathrm{~K} \mathrm{~s}^{-1}$. However, the maximum 
value of $d_{\mathrm{L}}$ decreases with increasing $\dot{T}$. At $\dot{T}=10$ and $20 \mathrm{~K} \mathrm{~s}^{-1}$, moreover, $d_{\mathrm{L}}$ decreases with further decreases in $V_{\mathrm{T}}$ in the discontinuous growth region. This behavior is explained by the fact that the mobility of FCRB is significantly reduced when $G$ is large according to Eq. $[1]$.

The short axis diameter, $d_{\mathrm{S}}$, versus $V_{\mathrm{T}}$ is shown in Figure $3(\mathrm{~b})$. One can see that the peak of $d_{\mathrm{S}}$ appears in the vicinity of $V_{\text {critical }}$ in the discontinuous growth region. In all the cases, $d_{\mathrm{S}}$ increases as $V_{\mathrm{T}}$ decreases to $V_{\text {critical }}$ in the continuous growth region and, after reaching the maximum value, it decreases with decreasing $V_{\mathrm{T}}$ in the discontinuous growth region. The peak value of $d_{\mathrm{S}}$ is reduced by increasing $\dot{T}$. Taking the behavior of $d_{\mathrm{L}}$ into account, one can readily comprehend that the coarsest grain structure emerges in the vicinity of $V_{\text {critical }}$ by the discontinuous grain growth. The reason for the peak of $d_{\mathrm{S}}$ to appear will be discussed in Section III. C.

Effects of initial short axis diameter, $d_{\text {initial }}$, on $d_{\mathrm{L}}$ and $d_{\mathrm{S}}$ are shown in Figure 4 . These are the results for $T_{\gamma}=1743 \mathrm{~K}, a_{\text {initial }}=3$ and $\dot{T}=1.0 \mathrm{~K} \mathrm{~s}^{-1} . V_{\text {critical }}$ decreases with increase in $d_{\text {initial }}$ (Eq. [1]). The sharp rising of $d_{\mathrm{L}}$ and the peak of $d_{\mathrm{S}}$ accordingly appear at the lower velocity for the larger $d_{\text {initial }}$. Importantly, the larger value of $d_{\text {initial }}$ leads to the larger peak value of $d_{\mathrm{S}}$, i.e., coarser grains. In the light of $V_{\text {critical }}, d_{\text {initial }}$ should be controlled to be large to avoid CCG formation, because the range of cooling conditions for discontinuous growth becomes narrow in the structure map. ${ }^{[12]}$ As is realized in Figure 4, however, the large value of 
$d_{\text {initial }}$ causes the formation of coarse grain structure once the discontinuous grain growth arises.

Figure 5 demonstrates effects of $a_{\text {initial }}$ and $T_{\gamma}$ calculated for $d_{\text {initial }}=150 \mu \mathrm{m}$ and $\dot{T}=$ $1.0 \mathrm{~K} \mathrm{~s}^{-1}$. It is noticed that $V_{\text {critical }}$ is independent of $a_{\text {initial, }}$, while it depends on $T_{\gamma}$, which can be seen in the sharp rising of $d_{\mathrm{L}}$ appearing at the lower velocity for the lower $T_{\gamma}$. Importantly, the peak value of $d_{\mathrm{S}}$ decreases with decrease in $T_{\gamma}$, which is in contrast to the dependence on $d_{\text {initial }}$ shown in Figure 4. Therefore, lowering $T_{\gamma}$ is quite effective both in preventing formation of CCG and in reducing the size of CCG. It is also important to notice that $d_{\mathrm{S}}$ for $a_{\text {initial }}=1$ is always larger than that for $a_{\text {initial }}=3$ in the discontinuous growth region. Namely, initial structures with equiaxed grains cause formation of coarser grains by the discontinuous growth, compared to those with columnar grains.

As shown in this section, the long axis diameter in final structure, $d_{\mathrm{L}}$, shows the sharp rising when the mechanism of grain growth changes from continuous to discontinuous mode. This is natural consequence because most of grains are elongated along $y$-direction from the lower edge to upper edge of $y$-axis in the final structure after the discontinuous growth. The short axis diameter, $d_{\mathrm{S}}$, exhibits the peak in the vicinity of the critical condition for discontinuous growth. The peak value of $d_{\mathrm{S}}$ largely depends on the cooling rate, $\dot{T}$ (thus the temperature gradient, $G$ ), initial short axis diameter, $d_{\text {initial }}$, initial aspect ratio, $a_{\text {initial }}$ and the temperature for completion of transformation to $\gamma$ single phase, $T_{\gamma}$. These dependencies have 
not been revealed in early works on as-cast $\gamma$ grains during casting. ${ }^{[5-8]}$ In the following section, these dependencies of $d_{\mathrm{S}}$ are discussed based on a theory of grain growth.

\section{Description of grain size based on a theory of grain growth}

For the sake of simplicity, we shall focus on the initial structure composed of equiaxed grains, i.e., $a_{\text {initial }}=1$. The dependences of $d_{\mathrm{S}}$ on growth conditions for $a_{\text {initial }}=1$ are essentially identical with those for $a_{\text {initial }}=3$, except that $d_{\mathrm{S}}$ for $a_{\text {initial }}=1$ is always larger than that for $a_{\text {initial }}=3$ in the discontinuous growth region (Figure 5(b)). The effect of $a_{\text {initial }}$ will be discussed later. First we consider the continuous grain growth.

Hillert developed a theory of grain growth. ${ }^{[17]} \mathrm{He}$ demonstrated that the time evolution of a critical grain radius, $r_{c}$, is described by $d r_{c}{ }^{2} / d t=\frac{1}{2} \alpha m \sigma$ ( Eq.[20] of Ref.[17] ) where $\alpha$ is a dimensionless constant depending on the dimensionality of system and $m$ was called mobility of the grain boundary and $\sigma$ is the grain boundary energy. $r_{c}$ is the critical radius below which the grain shrinks and is related to the average grain radius $\langle r\rangle$ as given by $r_{c}=g<r>$ with $g=1$ for 2-D and 9/8 for 3-D system. From this relation, the time evolution of average grain size $<r>$ in isothermal grain growth can be described by,

$$
\langle r(t)\rangle^{2}-\langle r(t=0)\rangle^{2}=\frac{1}{2} M(T) \cdot t
$$

where $\langle r(t=0)>$ is the averaged value of initial grain radius and $M(T)$ is given by $M(T)=\alpha m \sigma / g^{2}$. In this study, $M(T)$ is called the grain boundary mobility and it is related to 
$M^{\prime}(T)$ in Eq. [1] as $M(T)=M^{\prime}(T) / g^{2} \cdot{ }^{[12]}$ The temperature dependence of $M(T)$ is introduced in Eq. [3] and is given as $M(T)=M_{0} \exp (-Q /(R T))$ with a constant $M_{0}$ as commonly done in the experimental work. ${ }^{[7]}$ If a continuous cooling process is considered, Eq. [3] can be rewritten as,

$$
\langle r(t)\rangle^{2}-\langle r(t=0)\rangle^{2}=\frac{1}{2} \int_{0}^{t} M\left(T\left(t^{\prime}\right)\right) \cdot d t^{\prime}
$$

Note that the application of Eq. [4] to the continuous growth of our interest is not readily validated because the influence of temperature gradient on the grain growth was not explicitly considered in the derivation of Eq. [4]. In order to clarify the influence of temperature gradient, we performed additional simulations explained below.

We carried out the phase field simulations of grain growth under a fixed temperature distribution without cooling. The initial structure is equiaxed grain structure characterized by $a_{\text {initial }}=1$ and $d_{\text {initial }}=150 \mu \mathrm{m}$. A linear temperature gradient was applied along $y$-direction. The maximum temperature was fixed at $1743 \mathrm{~K}$ at the upper edge of $y$-axis, viz., $y=5 \mathrm{~mm}$. The cooling rate, $\dot{T}$, was set to zero. The grain growth starts at $t=0$ over the entire system but the growth rate of grains depends on the local temperature. After holding for $30 \mathrm{~s}$, the grain sizes were averaged over $x$-axis at each $y$-coordinate. The results for three different values of $G$ are shown in Figure 6. Although the data for large diameter are scattered, the grain size gradually increases with increase in $y$ in all the cases. The curves in Figure 6 are the results of Eq. [3] calculated at each $y$-coordinate with the assumption that the growing grains 
does not interact with each other along $y$-direction. Namely, these were obtained at each $y$-coordinate just by employing the corresponding local temperature in Eq. [3] with a value of $M_{0}$ estimated by isothermal phase field simulations. One can see that the calculated results of Eq. (3) are in good agreement with the results of simulations. This fact indicates that the grain growth under temperature gradient is described as the time evolution of assembly of the grains growing isothermally at the corresponding local temperature. This is consistent with the finding in early Monte Carlo study. ${ }^{[18]}$

The results of Figure 6 indicate that the temperature gradient, $G$, itself does not directly influence the grain growth, but the local temperature and the local cooling rate essentially control the grain growth kinetics. This fact verifies the applicability of Eq. [4] to the grain growth in the continuous growth region discussed in the previous section. This can be done by modifying Eq. [4] as follows,

$$
\left\langle r\left(y, t_{\mathrm{f}}\right)\right\rangle^{2}-\langle r(y, t=0)\rangle^{2}=-\frac{1}{2 \dot{T}} \int_{T_{\gamma}}^{T_{\mathrm{f}}(y)} M(T) \cdot d T
$$

where $<r\left(y, t_{\mathrm{f}}\right)>$ represents the grain radius averaged over $x$-axis at a given position, $y$, at the end of process $\left(t=t_{\mathrm{f}}\right) . T_{\mathrm{f}}(y)$ is the final temperature at a given position, $y$, as given by

$$
T_{\mathrm{f}}(y)=T_{\gamma}-G\left(y_{\max }-y\right), \quad[6]
$$

where $y_{\max }=5 \mathrm{~mm}$. The short axis diameters were calculated at each $y$-coordinate from Eq. [5] under a given cooling condition. The averaged values over $y$-coordinate calculated for different cooling conditions are shown in Figure 7 where the results of phase field simulations 
for $a_{\text {initial }}=1$ are also plotted. One can see that Eq. [5] successfully explains $d_{\mathrm{S}}$ simulated in the continuous growth region for different growth conditions.

As described in the introduction, in early works, the prediction of as-cast $\gamma$ grain size during continuous casting processes has been attempted based on a model of curvature-driven growth with the assumption that continuous grain growth takes place. ${ }^{[5-8]}$ It is important to note that the influence of temperature gradient on $\gamma$ grain growth was not explicitly discussed in these works and, in this regard, the applicability of early developed models to continuous casting process has not been sufficiently verified. On the other hand, the early developed models are essentially equivalent to Eqs. [4] and [5]. Hence, the present analysis demonstrates the validity of the early developed models for prediction of $\gamma$ grain size in continuous casting processes, as long as the continuous growth is dominant in the formation of as-cast $\gamma$ grain structure. As amply described, however, the CCG formation is attributed to the discontinuous growth. In Figure $7, d_{\mathrm{S}}$ obtained by the phase-field simulations largely deviate from the result of Eq. [5] in the vicinity of $V_{\text {critical }}$ where the discontinuous growth arises. Next we focus on the discontinuous growth region and attempt to describe the upper limit of $\gamma$ grain size near $V_{\text {critical. }}$

Shown in Figure 8(a) is the microstructural evolution process during cooling at $\dot{T}=$ $1.0 \mathrm{~K} \mathrm{~s}^{-1}$ and $G=1.2 \times 10^{4} \mathrm{~K} \mathrm{~m}^{-1}$ starting from initial equiaxed grain structure with $d_{\text {initial }}=150$ $\mu \mathrm{m}$. This cooling condition is in the discontinuous growth region and $V_{\mathrm{T}}$ is very close to 
$V_{\text {critical. }}$ The discontinuous growth takes place and the width of CCG gradually increases with the migration of FCRB. Importantly, the increase in width of CCG at the growth front of CCG (i.e., near FCRB) occurs by competitive growth between the grains near FCRB. When the tip radius of CCG at the growth front, $r^{\prime}$, is concerned, the time evolution of $r^{\prime}$ can be assumed to originate from normal grain growth between the grains of $r$ ' whose center positions move at a velocity of $V_{\mathrm{T}}$. This competitive growth at the growth front should proceed almost isothermally at a local temperature near FCRB. Hence, the grain growth at the growth front of CCG can be assumed to obey the relation, $d\left\langle r^{\prime}\right\rangle^{2} / d t=\frac{1}{2} M\left(T_{\gamma}^{\prime}\right)$ at each position just behind the FCRB. Here $T_{\gamma}^{\prime}$ is a local temperature at which the isothermal growth occurs and it should be close to $T_{\gamma}$. When $T_{\gamma}^{\prime}$ is a constant, the grain size at a given position, $y$, where the growth front passed through is represented by $\left\langle r^{\prime}(y)\right\rangle^{2}-\langle r(y=0)\rangle^{2}=\frac{1}{2} M\left(T_{\gamma}^{\prime}\right) \cdot t_{\mathrm{y}}$ as is similar to Eq. [3]. Here $t_{y}$ is the time at which the FCRB reaches the position $y$ and $\langle r(y=0)>$ is the initial radius at $y=0$. In addition to this growth process at the growth front, there is the grain growth behind the FCRB during cooling below $T_{\gamma}$. The initial grain size for this process corresponds to $\left\langle r^{\prime}(y)\right\rangle$ at each position. Then, the time evolution of the short axis radius of CCG can be approximated by the following equation,

$$
\langle r(y, t)\rangle^{2}-\langle r(y=0)\rangle^{2}=\frac{1}{2} M\left(T_{\gamma}^{\prime}\right) \cdot t_{\mathrm{y}}+\frac{1}{2} \int_{t_{\mathrm{y}}}^{t} M\left(T\left(t^{\prime}\right)\right) d t^{\prime} .
$$

The first term on the right-hand side of Eq. [7] represents the competitive growth between CCG at the growth front and the second term describes the grain growth during cooling below 
$T_{\gamma}$, which is equivalent to the right-hand side of Eq. [4]. Therefore, when the time evolution of grain size at a fixed position, $y$, is considered, the grain size discontinuously changes from its initial value (short axis diameter of FCG) to $\left\langle r^{\prime}(y)\right\rangle=\left(\langle r(y=0)\rangle^{2}+\frac{1}{2} M^{\prime}\left(T_{\gamma}^{\prime}\right) \cdot t_{\mathrm{y}}\right)^{1 / 2}$ at $t=t_{\mathrm{y}}$ and then the grain size gradually increases with time during the cooling below $T_{\gamma}$. The expression for Eq. [7] is applied to $t \geq t_{\mathrm{y}}$.

When $V_{\mathrm{T}} \leq V_{\text {critical }}$ is held, the discontinuous growth dominantly occurs. It should be noted that $V_{\text {critical }}$ given in Eq.(1) represents the velocity of shrinkage of FCG with average diameter, $d_{\text {initial. }}{ }^{[12]}$ Hence, when $V_{\mathrm{T}}=V_{\text {critical }}$ is satisfied, most of FCG shrink with migration of $T_{\gamma}$ position. However, some FCG with the short axis diameter larger than $d_{\text {initial }}$ cannot always shrink together with the migration of $T_{\gamma}$ position and they can shrink or grow, competing with the CCG. Since the discontinuous growth is dominant over the continuous growth from FCG when $V_{\mathrm{T}} \leq V_{\text {critical }}$, the final structure consists of fully columnar grains. Therefore, when $V_{\mathrm{T}}$ is very close to but less than $V_{\text {critical }}$, the discontinuous growth is basically dominant but the continuous growth from FCG partly occurs, which should hinder the competitive growth between CCG at the growth front described by the first term of Eq. [7] and should result in $d_{\mathrm{S}}$ smaller than the value predicted by Eq. [7]. As $V_{\mathrm{T}}$ decreases, the contribution of continuous growth from FCG diminishes and then the grain size should approach the value of Eq. [7]. However, further decrease in $V_{\mathrm{T}}$ causes reduction of width of CCG, as discussed in detail later. Therefore, Eq. [7] should provide a maximum value of $d_{\mathrm{S}}$ in 
the discontinuous growth region. The calculated results of Eq. [7] are shown in Figure 7. Since the mobility of the grain boundary at the growth front should be affected by a low temperature region when $G$ is high, we assumed that $T_{\gamma}^{\prime}=T_{\gamma}-G<r^{\prime}>$ in this calculation. Eq. [7] successfully describes the upper limit of $\gamma$ grain size by the discontinuous growth for different values of $\dot{T}, d_{\text {initial }}$ and $T_{\gamma}$. Note that $d_{\mathrm{S}}$ calculated by Eq. [7] decreases with decreasing $V_{\mathrm{T}}$ in the discontinuous growth region in Figure $7(\mathrm{~b})$. This is because $T_{\gamma}^{\prime}$ becomes low in this region and hence the contribution of the first term in Eq. [7] becomes small.

In Figure $7, d_{\mathrm{S}}$ obtained by the phase field simulations is consistent with the results of Eq. [5] in the continuous growth region. When $V_{\mathrm{T}}$ falls below $V_{\text {critical }}, d_{\mathrm{S}}$ by simulations sharply rise to the value predicted by Eq. [7] in the discontinuous growth region. However, $d_{\mathrm{S}}$ obtained by the simulations decreases with further decreases in $V_{\mathrm{T}}$, deviating from the result of Eq. [7] in all the cases. The reason for this behavior is discussed. Shown in Figure 8(b) is the microstructural evolution process for $G=4.0 \times 10^{4} \mathrm{~K} \mathrm{~m}^{-1}$. The other conditions are the same as those in Figure 8(a). Since $G$ is larger, $V_{\mathrm{T}}$ in Figure 8(b) is lower than that in Figure 8(a). Compared to Figure 8(a), the following differences appear in Figure 8(b). All the FCG shrink with the migration of FCRB. The width of CCG $\left(d_{\mathrm{S}}\right)$ is small. The FCRB is almost linear shape. The grain boundaries between CCG are linearly aligned almost parallel to $y$-direction. These features stem from the low value of $V_{\mathrm{T}}$. When $V_{\mathrm{T}}$ is low, the FCRB moves 
slowly. The FCRB is accordingly allowed to take an energetically favorable shape, which is linear shape because it minimizes the total grain boundary energy associated with FCRB. Furthermore, low $V_{\mathrm{T}}$ allows the grain boundaries between CCG to change their shape to linear shape in order to minimize the total grain boundary energy of CCG. Hence, the grain boundaries between CCG are linearly aligned along the moving direction of FCRB (y-direction). Then, these boundaries cannot move in 2D system because of no driving force for curvature-driven motion and, therefore, the increase in width of CCG does not occur. As a result, the width of CCG in Figure 8(b) is smaller than that in Figure 8(a). As $V_{\mathrm{T}}$ decreases, the FCRB moves more slowly and the grain boundaries become linear shape for a shorter period of time. The width of CCG should be accordingly smaller. Although we cannot provide a quantitative discussion about this process, this is the reason that $d_{\mathrm{S}}$ obtained by the phase field simulations decreases with decreasing $V_{\mathrm{T}}$ in the discontinuous growth region in Figures 3-5 and 7.

As discussed above, the growth of CCG along its short axis direction is retarded once the grain boundaries between CCG are linearly aligned along the moving direction of FCRB. When the initial grain structure is columnar shape, the linear alignment of boundaries appears in the early period of growth process. In the discontinuous growth region, therefore, the width of CCG in final structure for $a_{\text {initial }}=3$ is smaller than that for $a_{\text {initial }}=1$ as demonstrated in Figure 5(b). 
A quantitatively precise determination of grain size in 3-D system requires extensive computational resources and, therefore, our discussion has been limited to 2-D system. However, Eqs. [5] and [7] should be applicable to 3-D system when the grain boundary mobility, $M(T)$, is modified for 3-D case, more specifically, it is made roughly twice as large as the value of 2-D system. ${ }^{[12,17]}$ The difference between 2-D and 3-D systems appears in the low $V_{\mathrm{T}}$ region of discontinuous growth. As mentioned, when the grain boundary becomes linear shape, it does not move according to the curvature-driven motion in 2-D system, which is the reason that $d_{\mathrm{S}}$ decreases with decrease in $V_{\mathrm{T}}$ in the low $V_{\mathrm{T}}$ region of discontinuous growth. However, the linear grain boundary parallel to the growth direction of columnar grains in 2-D system should correspond to a curved grain boundary in 3-D system with a finite principal curvature of which the direction is almost normal to the growth direction. Hence, the CCG in 3-D system can grow along its short axis direction even when $V_{\mathrm{T}}$ is extremely low. In this regard, correction should be needed for the behavior of $d_{\mathrm{S}}$ in 3-D system in the low $V_{\mathrm{T}}$ region. This point remains to be clarified in detail in a future work.

\section{Comparison with the experimental data}

The recent experimental investigation revealed that the width of CCG increases only

near FCRB, viz., near $T_{\gamma}$ and it does not virtually increases during cooling below $T_{\gamma}{ }^{[11]}$ This fact is in marked contrast to the discussions in early works on $\gamma$ grain growth during casting. 
In our previous report, ${ }^{[11]}$ we considered that the increase in CCG width near $T_{\gamma}$ could be explained by 2-D grain growth on the plane perpendicular to the moving direction of FCRB because of their columnar shape. As discussed in the previous section, however, such an increase in CCG width should originate from 3-D growth process between grains near FCRB, the contribution of which is described by the first term of Eq. [7]. In this section, we carry out a comparison between the experimental data obtained in our previous study ${ }^{[11]}$ and the calculated result in order to check the validity of the present analysis on one hand and in order to quantitatively ascertain the experimental fact ${ }^{[11]}$ on the other hand.

In the previous study on rapid unidirectional solidification, ${ }^{[11]}$ the as-cast $\gamma$ grain structure of Fe- 0.2 mass $\% \mathrm{C}-0.2 \mathrm{mass} \% \mathrm{Si}-0.8 \mathrm{mass} \% \mathrm{Mn}$ steel $(0.2 \mathrm{C}$ steel) consisted of CCG over the entire region of sample. Hence, it is expected that the condition of $V_{\mathrm{T}} \leq V_{\text {critical }}$ (Eq. [1]) was always satisfied during the casting process. From the cooling curves measured at several distances from the mold wall, the thermal velocity $V_{\mathrm{T}}$ during casting of $0.2 \mathrm{C}$ steel was estimated. ${ }^{[11]}$ The result is plotted with respect to the distance from the mold wall in Figure 9 (a). Although slightly scattered, $V_{\mathrm{T}}$ falls within a relatively narrow range of $2 \times 10^{-4}-6 \times 10^{-4} \mathrm{~m}$ $\mathrm{s}^{-1}$. The measured $V_{\mathrm{T}}$ cannot be compared with $V_{\text {critical }}$ shown in Figures 3-5 and 7, because $V_{\text {critical }}$ in Figures 3-5 and 7 are the values for 2-D system with a constant $d_{\text {initial }}$ over the whole system. For comparison with the measured $V_{\mathrm{T}}, V_{\text {critical }}$ should be recalculated for 3-D system by taking dependence of $d_{\text {initial }}$ on the position into account. For this, we measured the short 
axis diameters of FCG near FCRB at several distances from the mold wall in the quenched samples. Also, we employed $T_{\gamma}=1751 \mathrm{~K},{ }^{[19]} M_{0}=4.0 \times 10^{-3} \mathrm{~m}^{2} \mathrm{~s}^{-1[7]}$ and $Q=177 \mathrm{~kJ} \mathrm{~mol}^{-1[7]}$ for $0.2 \mathrm{C}$ steel. The calculated values of $V_{\text {critical }}$ are shown in Figure $9(\mathrm{a})$. One can see that $V_{\text {critical }}$ is very close to $V_{\mathrm{T}}$ in the previous experiment. ${ }^{[11]}$ Since $V_{\text {critical }} \approx V_{\mathrm{T}}$ in Fig. 9(a), one cannot actually conclude which the growth mode, the discontinuous or continuous mode, is dominant in the casting experiment. As mentioned above, however, the as-cast $\gamma$ grain structure of $0.2 \mathrm{C}$ steel consisted of $\mathrm{CCG}$ over the entire region of sample ${ }^{[11]}$ and this fact indicates that the condition of $V_{\mathrm{T}} \leq V_{\text {critical }}$ was always satisfied during the casting process. Therefore, it is expected that measured $V_{\mathrm{T}}$ is always close to but less than $V_{\text {critical }}$. Then, the model given by Eq. [7] should be applicable to the description of size of CCG. In what follows, we analyze the grain size in terms of both the continuous (Eq. [5]) and the discontinuous modes (Eq. [7]).

The measured values of $d_{\mathrm{S}}$ of CCG in $0.2 \mathrm{C}$ steel cooled to room temperature ${ }^{[11]}$ are shown in Figure $9(\mathrm{~b}) . d_{\mathrm{S}}$ gradually increases with increase in the distance from the mold wall. The calculated results of Eq. [7] are indicated by filled circle symbols in Figure 9(b). These values were calculated by utilizing the cooling curves measured at each position. The cooling curves near the mold wall, viz., $x<8 \mathrm{~mm}$ were not employed because of their large uncertainties in measurement accuracy. In the recent experimental study ${ }^{[11]}$, it was found that the diameter of $\mathrm{CCG}$ at the growth front measured in the quenched sample was almost 
coincident with the grain diameter at the corresponding position (height) in the sample naturally cooed to room temperature. As for the initial diameter in the calculation of Eq. [7], therefore, we used $d_{\mathrm{S}}=0.46 \mathrm{~mm}$ measured at $1 \mathrm{~mm}$ away from the mold wall in the sample naturally cooled to room temperature. The calculation was accordingly started from $1 \mathrm{~mm}$ away from the mold wall. One can see that the result of Eq. [7] is in excellent agreement with the experimental data. It should be pointed out that initial aspect ratio of FCG is always larger than 1 in the experiment but Eq. [7] is in a strict sense valid for $a_{\text {initial }}=1$. In addition, there might be a certain uncertainty involved in the accuracy of $M(T)$ used in the present calculation. Although these points remain to be examined quantitatively, the agreement between the experimental and calculated results in Figure 9(b) support the validity of the model proposed in this study.

The half-filled open circles in Figure 9(b) represent the contribution of the first term in Eq. [7], viz., $d_{\mathrm{S}}$ calculated without the grain growth during cooling below $T_{\gamma}$. By comparison with the experimental data, it is evident that within the model of Eq. [7], the grain growth takes place mainly due to the competitive growth near FCRB and the contribution of grain growth during cooling below $T_{\gamma}$ is small. It should be noted that the main concern in early works on grain growth of as-cast $\gamma$ structure ${ }^{[5-8]}$ has been the cooling process below $T_{\gamma}$. In contrast to the early discussions, our model suggests that the cooling process below $T_{\gamma}$ should not be the dominant process in controlling as-cast $\gamma$ grain size during casting and the 
grain size should be mainly determined by the grain growth isothermally taking place near $T_{\gamma}$.

This is fully consistent with the recent experimental finding. ${ }^{[11]}$

The triangle plots in Figure 9(b) indicate the results for the continuous growth calculated by Eq. [4]. The measured cooling curve at each position was employed and the measured diameter of FCG at each position was used as the initial diameter at each position in the calculation. The other input parameters are the same as those used in the calculation of Eq. [7]. It is seen that the continuous growth model, Eq. [4], fairly underestimates the grain size. Therefore, the experimental results of the grain size cannot be explained based on the continuous grain growth mechanism.

Effects of the initial grain structure near the cast surface are discussed. The initial grain size near the cast surface affects the onset of discontinuous growth and thus the final grain size. If fine grains form near the mold side, the discontinuous growth process is prone to arise from such a fine structure near the mold wall because $V_{\text {critical }}$ given by Eq. [1] is high. Even if the grains growth does not sufficiently takes place due to high cooling rate near the mold side, the discontinuous growth should arise at a position where the grain growth is allowed and $V_{\mathrm{T}} \leq V_{\text {critical }}$ is realized ${ }^{[12]}$. In fact, as demonstrated elsewhere, ${ }^{[20]}$ when the carbon concentration is higher and thus $V_{\text {critical }}$ is relatively lower due to lowering of $T_{\gamma}$, fine equiaxed $\gamma$ grains are more likely to form in the vicinity of the mold wall and, however, the $\gamma$ grain structure changes to $\mathrm{CCG}$ at a certain distance from the mold wall. In the case of $0.2 \mathrm{C}$ steel of 
our focus, the CCG develop from the cast surface ${ }^{[11]}$ and, therefore, the discontinuous growth starts almost at the cast surface. The formation process of initial coarse $\gamma$ grains near the cast surface was not fully elucidated in the experimental work $^{[11]}$ and it was not explicitly addressed in the present analysis where the calculation of Eq. [7] was started from $1 \mathrm{~mm}$ away from the mold wall. The formation process of initial $\gamma$ grains near the cast surface remains to be clarified.

\section{CONCLUSIONS}

As-cast coarse columnar $\gamma$ grains (CCG) in peritectic carbon steels develop by the mechanism of discontinuous grain growth. When the migration velocity of $T_{\gamma}$ position, $V_{\mathrm{T}}$, decreases to the critical velocity, $V_{\text {critical}}$, the discontinuous grain growth occurs and the CCG structure accordingly forms. In this study, effects of growth conditions on as-cast $\gamma$ grain size were scrutinized by means of 2-D phase field simulations. It was demonstrated that the long axis diameter of as-cast $\gamma$ grain, $d_{\mathrm{L}}$, sharply increases when the mechanism of grain growth changes from continuous to discontinuous mode. Importantly, the dependence of the short axis diameter, $d_{\mathrm{S}}$, on $V_{\mathrm{T}}$ exhibits the peak near $V_{\text {critical }}$. Then, the dependences of $d_{\mathrm{S}}$ on growth conditions were discussed based on a theory of grain growth and a model for predicting the upper limit of $d_{\mathrm{S}}$ was put forward. The comparison between the calculated result and the experimental data ${ }^{[11]}$ supports the validity of this model. 
In the present study, we have not explicitly dealt with the formation of FCG structure.

Since the shape and size of FCG structure largely affect the final grain size, the detailed investigations on FCG formation should be one of the important subjects to be addressed for a further progress in controlling the as-cast $\gamma$ grain structure.

\section{ACKNOWLEDGMENTS}

This work was supported by $18^{\text {th }}$ ISIJ Research Promotion Grant and Grant-in-Aid for Young Scientists (A) (No. 2268067) from MEXT, Japan. One of us (M.O.) also acknowledges partial financial support from the Next Generation Super Computing Project, Nano-science Program, MEXT, Japan. 


\section{REFERENCES}

1. L. Schmidt and Å. Josefsson: Scand . J. Metall, 1974, vol. 3, pp. 193-99.

2. B. Mintz and J.M. Arrowsmith: Met. Technol., 1979, vol. 6, pp. 24-32.

3. Y. Maehara, K. Yasumoto, Y. Sugitani, K. Gunji: Trans. ISIJ, 1985, vol. 25, pp. 1045-52.

4. D.N. Crowther and B. Mintz: Mater. Sci. Technol., 1986, vol. 2, pp. 951-55.

5. N. Yoshida, O. Umezawa and K. Nagai: ISIJ Int., 2004, vol. 44, pp. 547-55.

6. H.S. Kim, Y. Kobayashi and K. Nagai: Acta Mater., 2006, vol. 54, pp. 2441-49.

7. C. Bernhard, J. Reiter and H. Presslinger: Metall. Mater. Trans. B, 2008, vol. 39B, pp. 885-95.

8. N. Yoshida, Y. Kobayashi and N. Nagai: Tetsu-toHagané, 2004, vol. 90 (2004).

9. V. Karlinski de Barcellos, V. Lopes da Silva Gschwenter, H. Kytönen, C. Alexandre dos Santos, J. Alvares Spim, S. Louhenkilpi and J. Miettinen: Steel Research Int., 2010, vol. 81, pp. $461-71$.

10. J. Reiter, C. Bernhard and H. Presslinger: Mater. Characterization, 2008, vol. 59, pp.737-46.

11. S. Tsuchiya, M. Ohno, K. Matsuura and K. Isobe: Acta Mater., 2011, vol. 59, pp.3334-42.

12. M. Ohno, S. Tsuchiya and K. Matsuura: Acta Mater., 2011, vol. 59, pp.5700-09.

13. I. Steinbach and F. Pezzola: Physica D, 1999, vol. 134, pp. 385-93.

14. S.G. Kim, D.I. Kim, W.T. Kim and Y.B. Park: Phys. Rev. E, 2006, vol. 74., pp. 
061605-1-14.

15. E.D. Hondros: Proc. R. Soc. London. A, 1965, vol. 286, pp. 479-98.

16. M. Apel, B. Böttger, J. Rudnizki, P. Schaffnit and I. Steinbach: ISIJ Int., 2009, Vol. 49, pp. 1024-29.

17. M. Hillert: Acta Metall., 1965, vol. 13, pp.227-38.

18. E.A. Holm, N. Zacharopoulos, D.J. Srolovitz: Acta Mater., 1998, vol. 46, pp.953-64.

19. CompuTherm LLC, http://www.computherm.com/ [accessed 08.09.10].

20. S. Tsuchiya, M. Ohno and K. Matsuura, in preparation. 


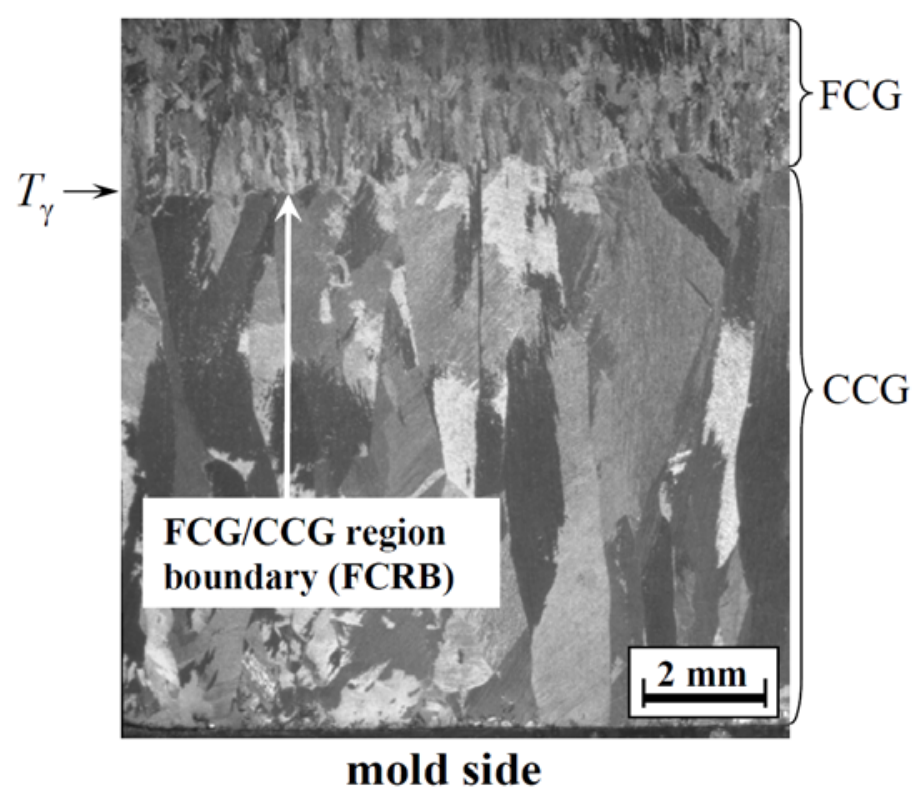

Fig. 1. Micrograph of $\gamma$ grain structure in solidifying sample of 0.2 mass $\%$ carbon steel quenched during unidirectional solidification in metallic mold casting. ${ }^{[10]}$ The solidification unidirectionally proceeds from the lower to upper part of the micrograph. The FCG region corresponds to liquid $+\gamma$ two phase field, while the CCG region is $\gamma$ single phase field. 
(a)

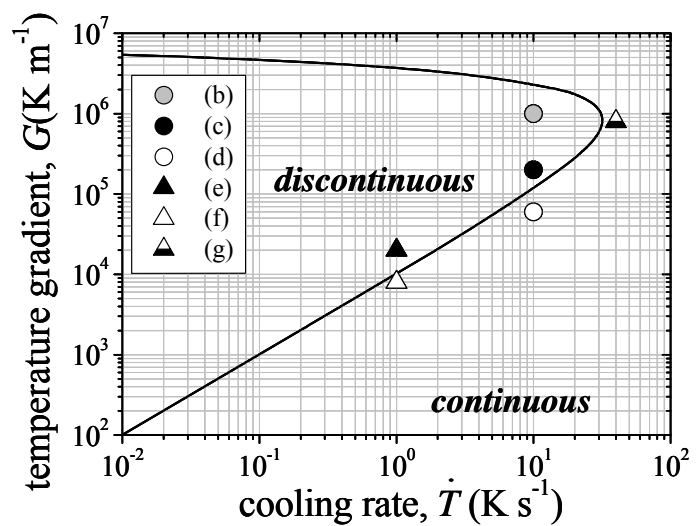

(b) $\dot{T}=10 \mathrm{~K} \mathrm{~s}^{-1}, \quad G=1.0 \times 10^{6} \mathrm{~K} \mathrm{~m}^{-1}$

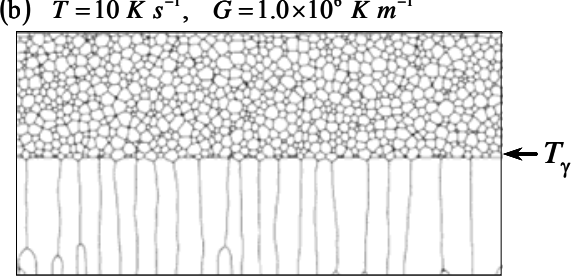

(c) $\dot{T}=10 \mathrm{~K} \mathrm{~s}^{-1}, \quad G=2.0 \times 10^{5} \mathrm{~K} \mathrm{~m}^{-1}$

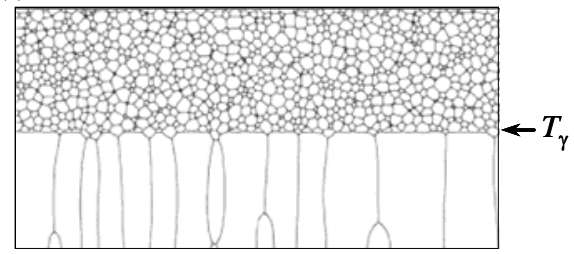

(d) $\dot{T}=10 \mathrm{~K} \mathrm{~s}^{-1}, \quad G=6.0 \times 10^{4} \mathrm{~K} \mathrm{~m}^{-1}$

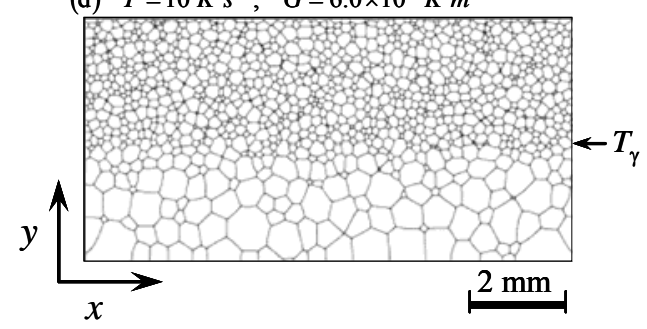

(e) $\dot{T}=1.0 \mathrm{~K} \mathrm{~s}^{-1}, \quad G=2.0 \times 10^{4} \mathrm{~K} \mathrm{~m}^{-1}$

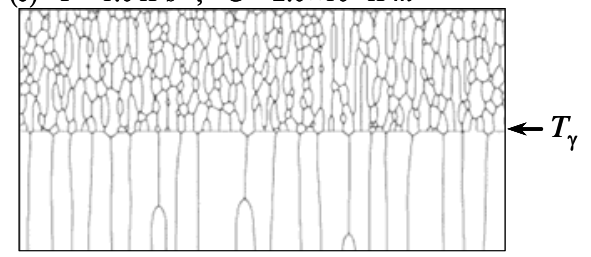

(f) $\dot{T}=1.0 \mathrm{~K} \mathrm{~s}^{-1}, \quad G=8.0 \times 10^{3} \mathrm{~K} \mathrm{~m}^{-1}$

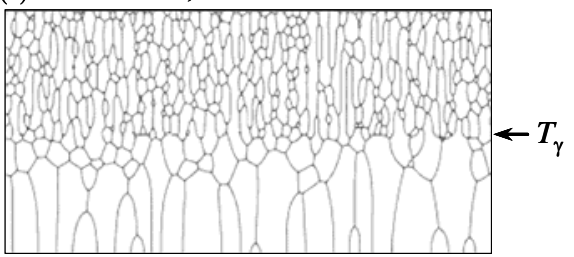

(g) $\dot{T}=40 \mathrm{~K} \mathrm{~s}^{-1}, \quad G=8.0 \times 10^{5} \mathrm{~K} \mathrm{~m}^{-1}$

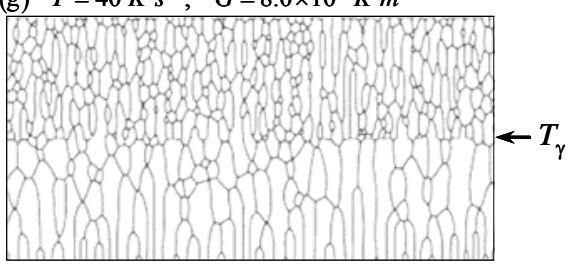

Fig. 2. Calculated results for $T_{\gamma}=1743 \mathrm{~K}$ and $d_{\text {initial }}=150 \mu \mathrm{m}$. (a) Structure map specifying range of cooling conditions for discontinuous grain growth. (b-g) Microstructures during cooling processes indicated in figure (a) at a time when the position of $T_{\gamma}$ reaches half of total length of $y$-axis. The position of $T_{\gamma}$ is indicated by the arrow on the right-hand side in each micrograph. The initial structures for (b)-(d) are the equiaxed grain structure with initial aspect ratio, $a_{\text {initial }}=1$, while those for $(\mathrm{e})-(\mathrm{g})$ are the columnar grain structure with $a_{\text {initial }}=3$. 
(a)

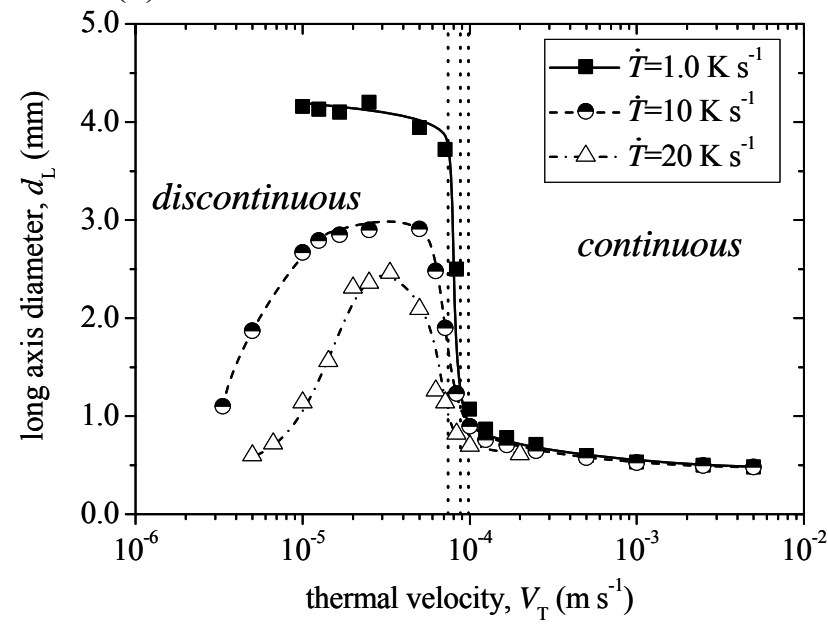

(b)

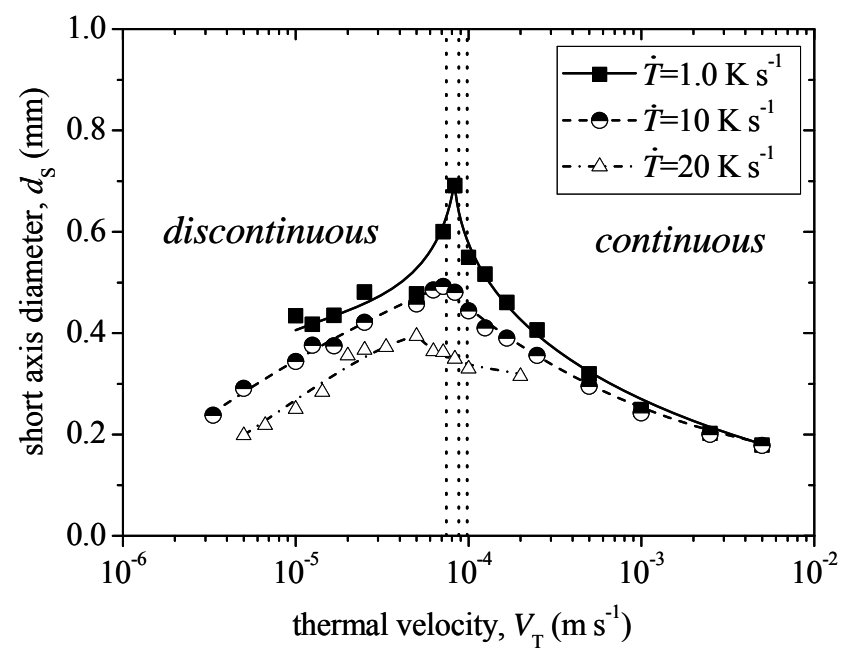

Fig. 3. Dependences of grain diameters on thermal velocity. (a) Long axis and (b) short axis diameters at three cooling rates calculated for $T_{\gamma}=1743 \mathrm{~K}, d_{\text {initial }}=150 \mu \mathrm{m}$ and $a_{\text {initial }}=3$. The vertical dotted lines indicate the critical velocities of discontinuous grain growth for three different cooling rates (right; $\dot{T}=1.0 \mathrm{~K} \mathrm{~s}^{-1}$, middle; $\dot{T}=10 \mathrm{~K} \mathrm{~s}^{-1}$, left; $\dot{T}=20 \mathrm{~K} \mathrm{~s}^{-1}$ ). 
(a)

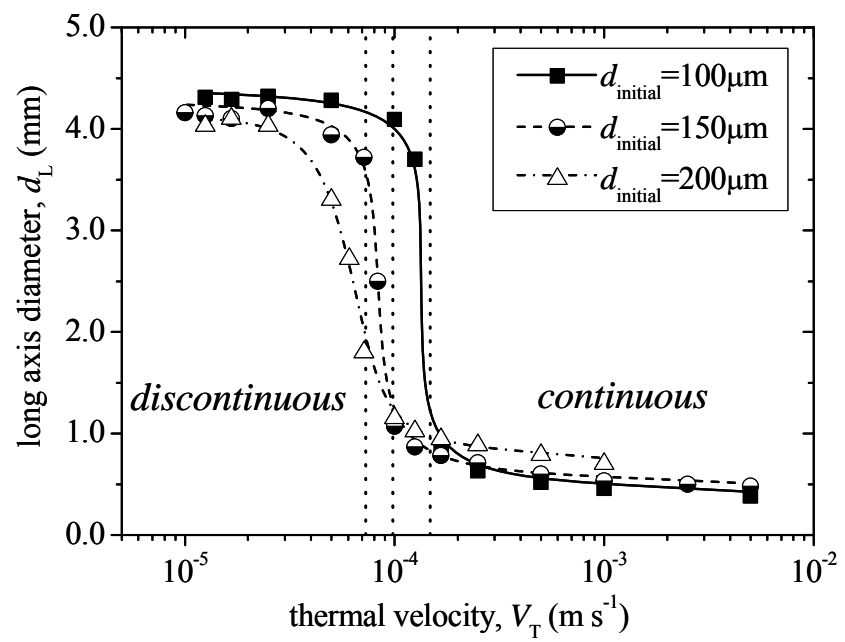

(b)

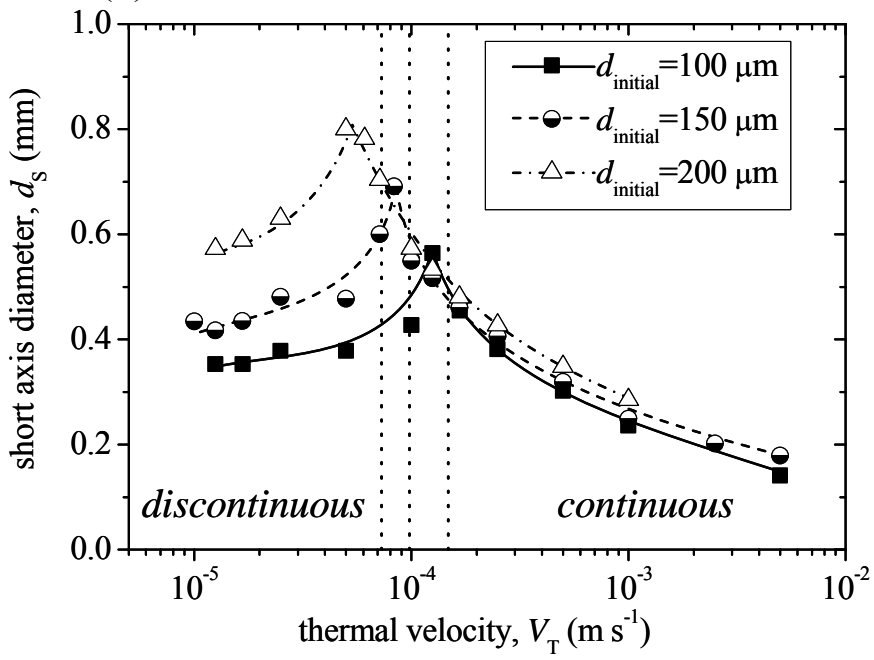

Fig. 4. Dependences of grain diameters on thermal velocity. (a) Long axis and (b) short axis diameters calculated for $a_{\text {initial }}=3, \dot{T}=1.0 \mathrm{~K} \mathrm{~s}^{-1}, T_{\gamma}=1743 \mathrm{~K}$ and three different values of $d_{\text {initial }}$. The vertical dotted lines indicate the critical velocities for three different $d_{\text {initial }}$ (right; $d_{\text {initial }}=100 \mu \mathrm{m}$, middle; $d_{\text {initial }}=150 \mu \mathrm{m}$, left; $\left.d_{\text {initial }}=200 \mu \mathrm{m}\right)$. 
(a)

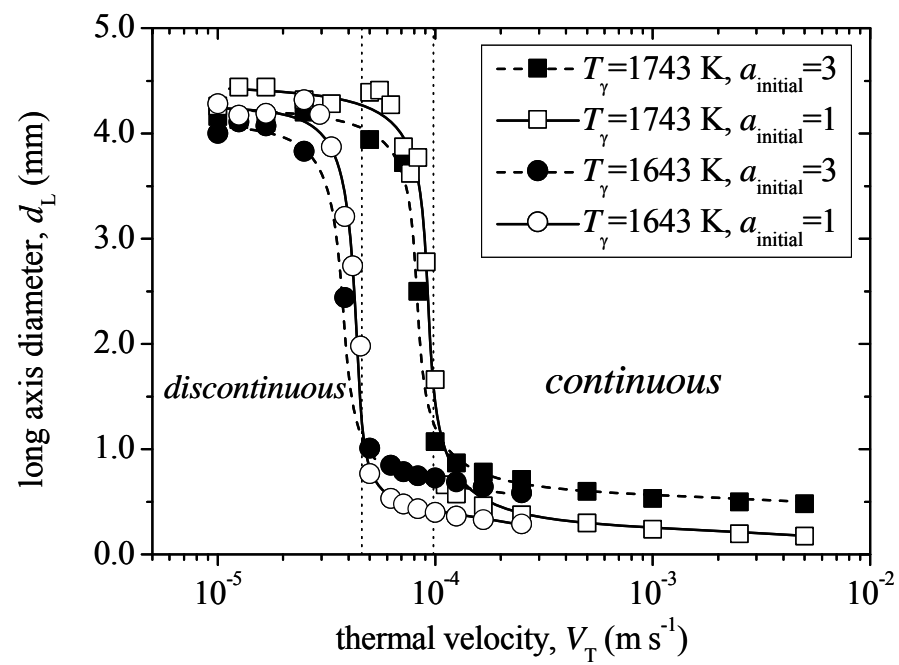

(b)

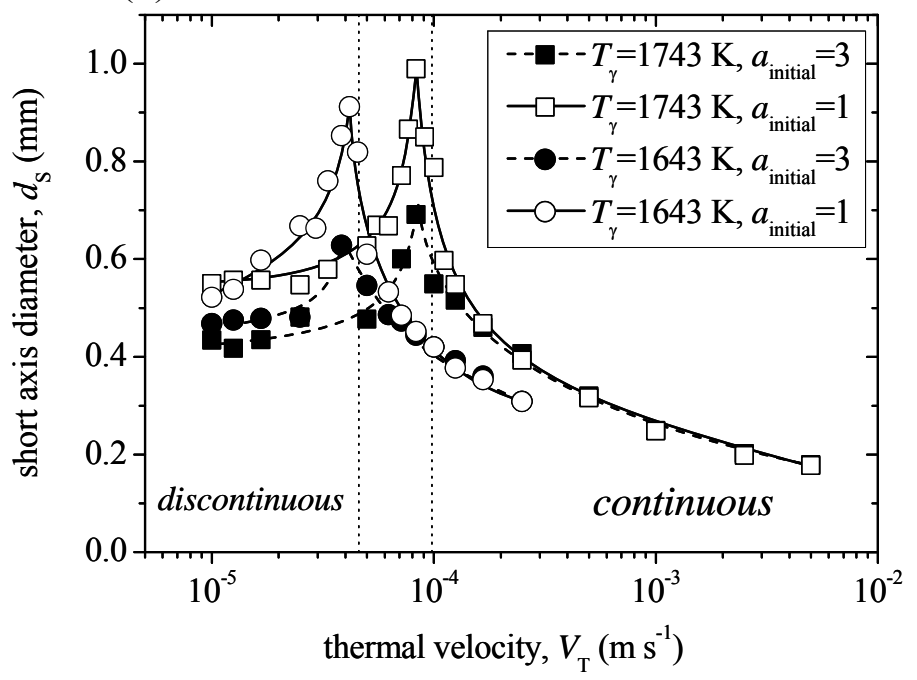

Fig. 5. Dependences of grain diameters on thermal velocity. (a) Long axis and (b) short axis diameters calculated for $d_{\text {initial }}=150 \mu \mathrm{m}$ and $\dot{T}=1.0 \mathrm{~K} \mathrm{~s}^{-1}$ and different values of $T_{\gamma}$ and $a_{\text {initial }}$. The vertical dotted lines indicate the critical velocities for $T_{\gamma}=1743 \mathrm{~K}$ (right) and $T_{\gamma}=$ $1643 \mathrm{~K}$ (left). 


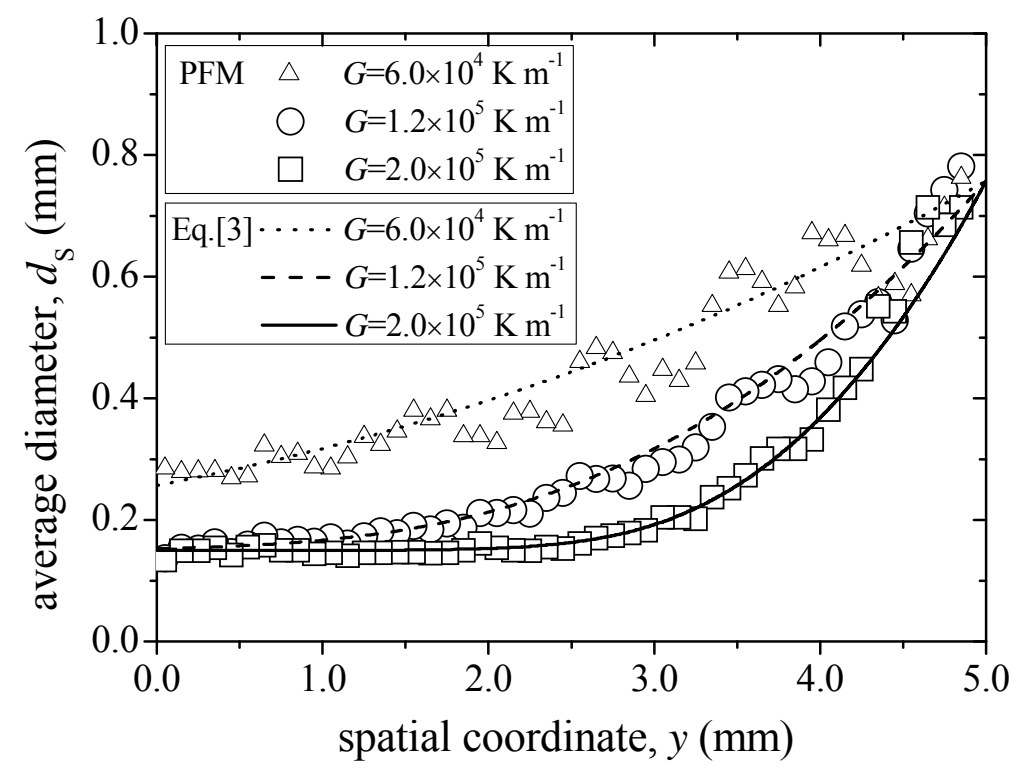

Fig. 6. Grain diameter averaged over $x$-coordinate during grain growth under a fixed temperature distribution. The results of the phase field simulations are compared with the results of Eq. [3]. The initial structure is the one with $a_{\text {initial }}=1$ and $d_{\text {initial }}=150 \mu \mathrm{m}$. 
(a) $T_{\gamma}=1743 \mathrm{~K}, d_{\text {initial }}=150 \mu \mathrm{m}, \dot{T}=1.0 \mathrm{~K} \mathrm{~s}^{-1}$

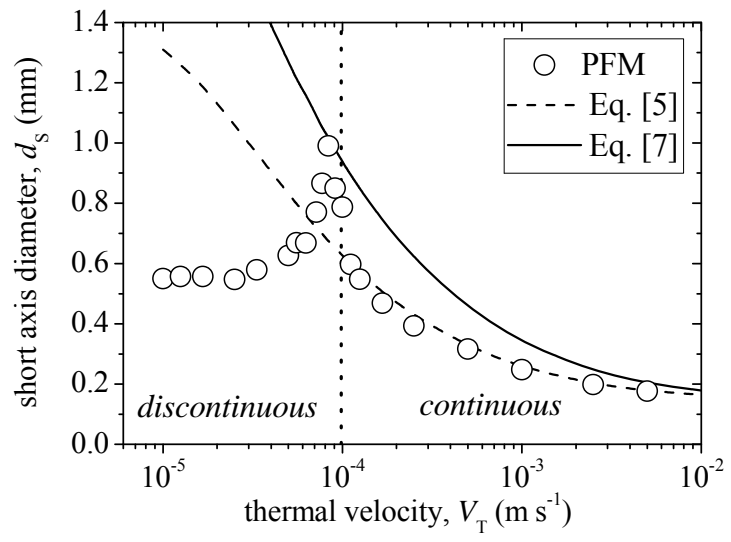

(c) $T_{\gamma}=1643 \mathrm{~K}, d_{\text {initial }}=150 \mu \mathrm{m}, \quad \dot{T}=1.0 \mathrm{~K} \mathrm{~s}^{-1}$

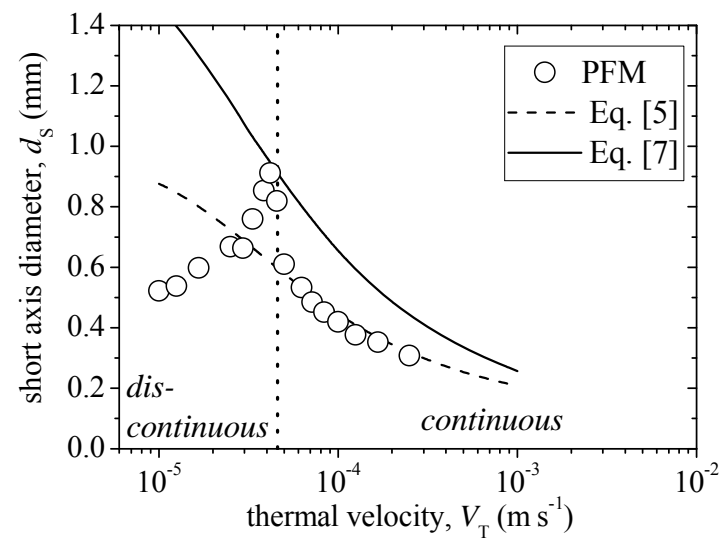

(b) $T_{\gamma}=1743 \mathrm{~K}, d_{\text {initial }}=150 \mu \mathrm{m}, \dot{T}=10 \mathrm{~K} \mathrm{~s}^{-1}$

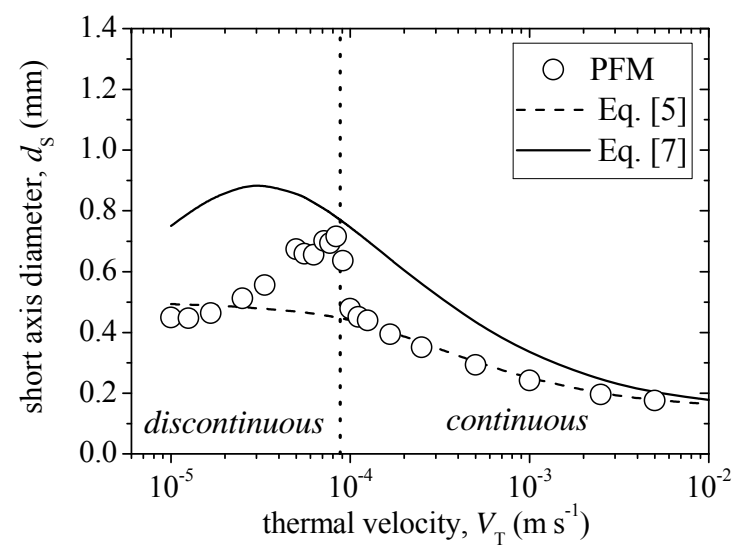

(d) $T_{\gamma}=1743 \mathrm{~K}, d_{\text {initial }}=200 \mu \mathrm{m}, \quad \dot{T}=1.0 \mathrm{~K} \mathrm{~s}^{-1}$

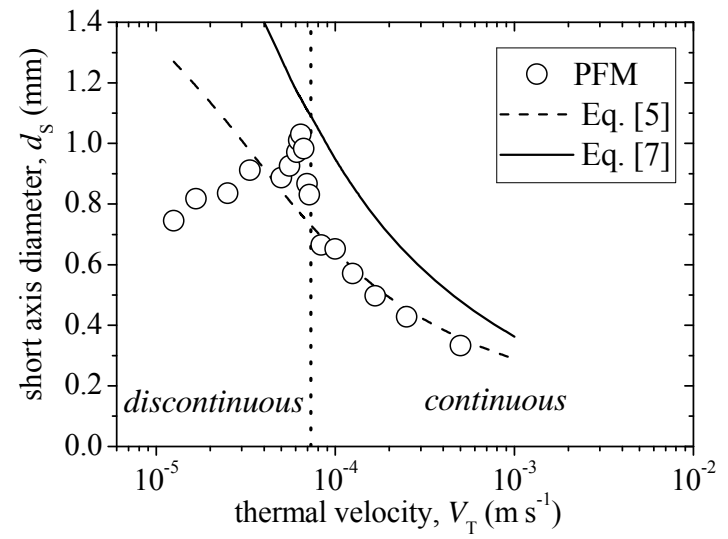

Fig. 7. Comparisons in short axis diameter between results of phase-field simulations, Eq. [5] and Eq. [7] for (a) $T_{\gamma}=1743 \mathrm{~K}, d_{\text {initial }}=150 \mu \mathrm{m}$ and $\dot{T}=1.0 \mathrm{~K} \mathrm{~s}^{-1}$, (b) $T_{\gamma}=1743 \mathrm{~K}, d_{\text {initial }}=$ $150 \mu \mathrm{m}$ and $\dot{T}=10 \mathrm{~K} \mathrm{~s}^{-1}$, (c) $T_{\gamma}=1643 \mathrm{~K}, d_{\text {initial }}=150 \mu \mathrm{m}$ and $\dot{T}=1.0 \mathrm{~K} \mathrm{~s}^{-1}$, (d) $T_{\gamma}=1743 \mathrm{~K}$, $d_{\text {initial }}=200 \mu \mathrm{m}$ and $\dot{T}=1.0 \mathrm{~K} \mathrm{~s}^{-1}$. The vertical dotted line in each figure represents $V_{\text {critical }}$. 


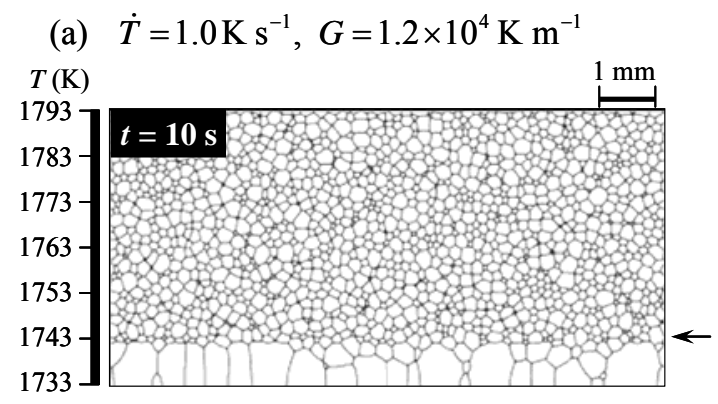

(b) $\dot{T}=1.0 \mathrm{~K} \mathrm{~s}^{-1}, G=4.0 \times 10^{4} \mathrm{~K} \mathrm{~m}^{-1}$
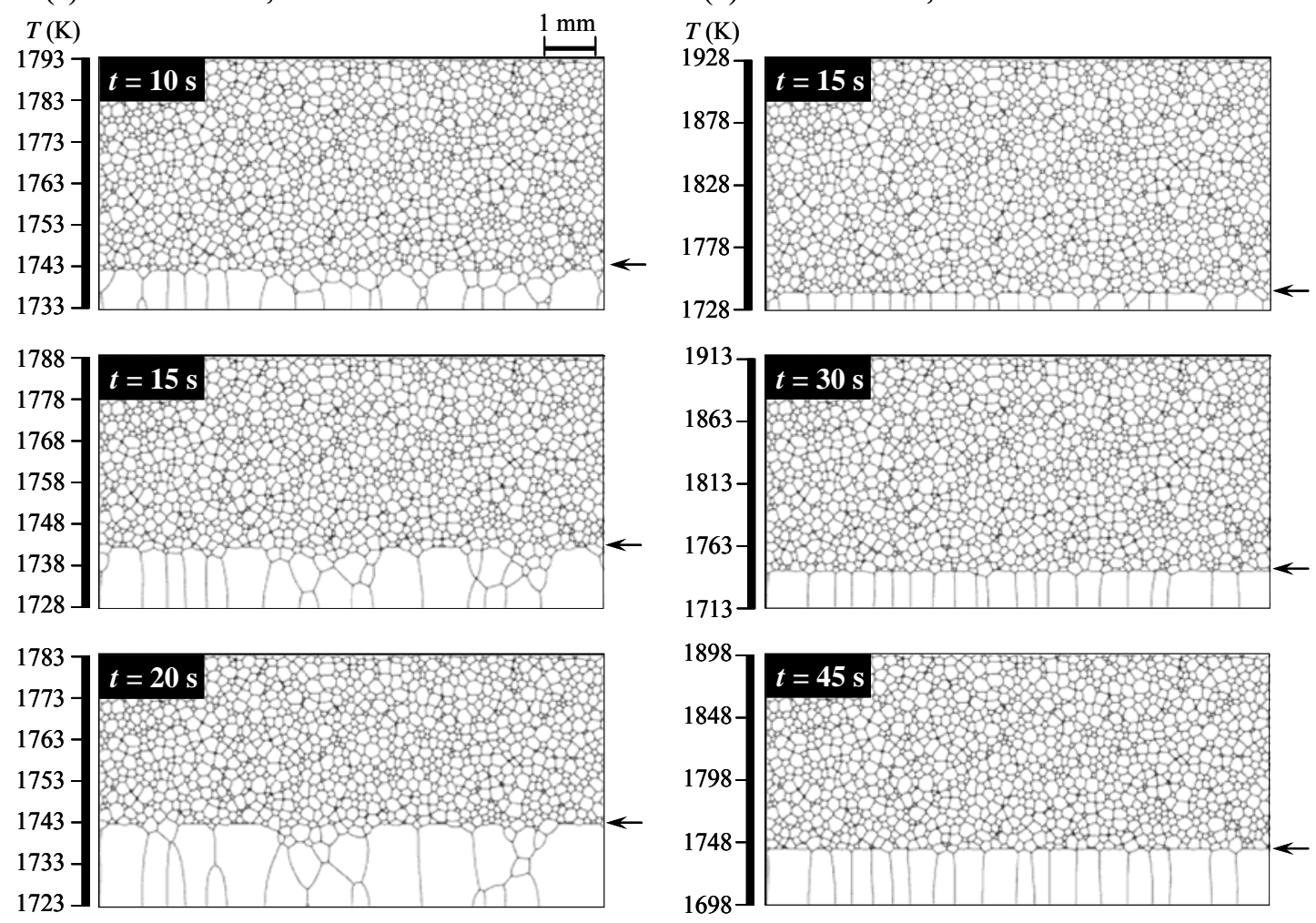

Fig. 8. Microstructural evolution processes for $T_{\gamma}=1743 \mathrm{~K}, a_{\text {initial }}=1$ and $d_{\text {initial }}=150 \mu \mathrm{m}$, (a) $\dot{T}=1.0 \mathrm{~K} \mathrm{~s}^{-1}$ and $G=1.2 \times 10^{4} \mathrm{~K} \mathrm{~m}^{-1}$, (b) $\dot{T}=1.0 \mathrm{~K} \mathrm{~s}^{-1}$ and $G=4.0 \times 10^{4} \mathrm{~K} \mathrm{~m}^{-1}$. The temperature is specified on the left-hand side and the position of $T_{\gamma}$ is indicated by the arrow on the right-hand side in each image. 
(a)

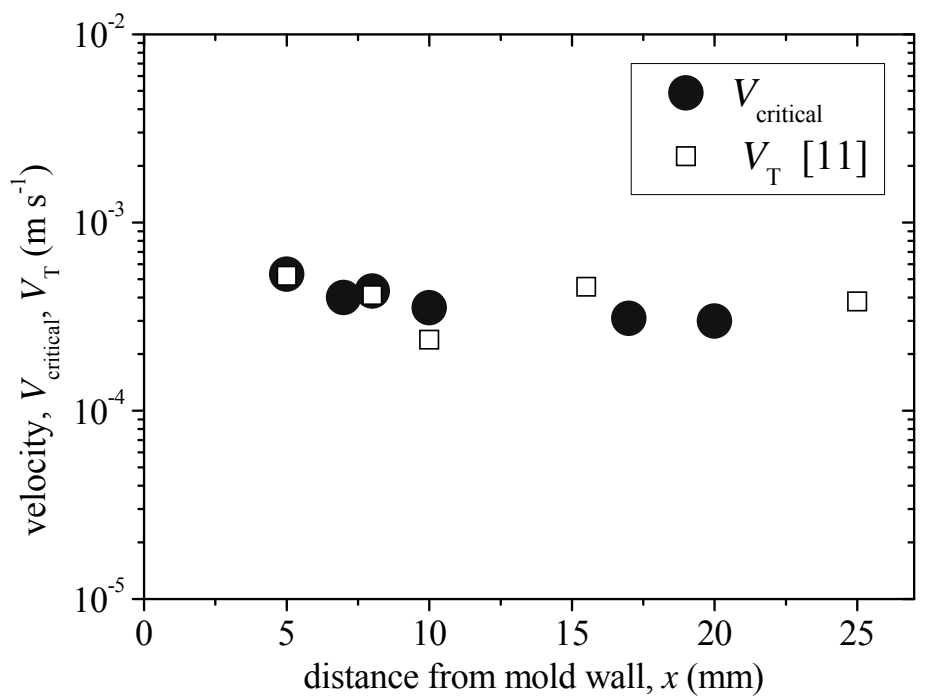

(b)

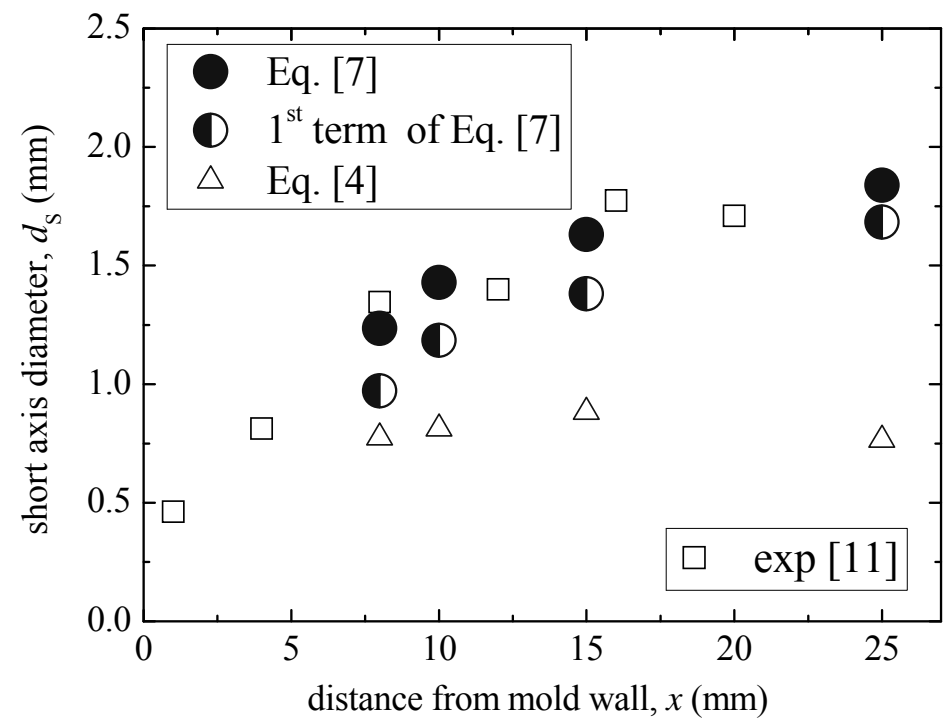

Fig. 9. Comparisons between the experimental data [11] and the calculated results. (a) Dependence of $V_{\mathrm{T}}$ measured in the previous study ${ }^{[11]}$ and $V_{\text {critical }}$ calculated by Eq. [1] on the distance from the mold wall. (b) Experimental and calculated results for short axis diameters with respect to distance from mold wall. 